\title{
Disruption of phytoene desaturase gene results in albino and dwarf phenotypes in Arabidopsis by impairing chlorophyll, carotenoid, and gibberellin biosynthesis
}

\author{
Genji Qin ${ }^{1}$, Hongya $\mathrm{Gu}^{1,2}$, Ligeng Ma ${ }^{1,3}$, Yiben Peng ${ }^{1}$, Xing Wang Deng ${ }^{1,3}$, Zhangliang Chen ${ }^{1}$, Li-Jia Qu ${ }^{1,2}$ \\ ${ }^{I}$ Peking-Yale Joint Center for Plant Molecular Genetics and Agrobiotechnology, National Laboratory for Protein Engineering and \\ Plant Genetic Engineering, College of Life Sciences, Peking University, Beijing 100871, China; ${ }^{2}$ The National Plant Gene Research \\ Center (Beijing), Beijing 100101, China; ${ }^{3}$ Department of Molecular, Cellular, and Developmental Biology, Yale University, New \\ Haven Connecticut 62520-8104, USA
}

Carotenoids play an important role in many physiological processes in plants and the phytoene desaturase gene (PDS3) encodes one of the important enzymes in the carotenoid biosynthesis pathway. Here we report the identification and analysis of a T-DNA insertion mutant of $P D S 3$ gene. Functional complementation confirmed that both the albino and dwarf phenotypes of the $p d s 3$ mutant resulted from functional disruption of the $P D S 3$ gene. Chloroplast development was arrested at the proplastid stage in the $p d s 3$ mutant. Further analysis showed that high level of phytoene was accumulated in the $p d s 3$ mutant. Addition of exogenous $\mathrm{GA}_{3}$ could partially rescue the dwarf phenotype, suggesting that the dwarf phenotype of the $p d s 3$ mutant might be due to GA deficiency. Microarray and RT-PCR analysis showed that disrupting $P D S 3$ gene resulted in gene expression changes involved in at least 20 metabolic pathways, including the inhibition of many genes in carotenoid, chlorophyll, and GA biosynthesis pathways. Our data suggest that the accumulated phytoene in the $p d s 3$ mutant might play an important role in certain negative feedbacks to affect gene expression of diverse cellular pathways.

Keywords: Arabidopsis thaliana, phytoene desaturase, albino, dwarf, microarray analysis

Cell Research (2007) 17:471-482. doi: 10.1038/cr.2007.40; published online 8 May 2007

\section{Introduction}

Carotenoids, the membrane-bound lipid-soluble pigments including carotene and xanthophyll, are a class of $\mathrm{C}_{40}$

Correspondence: Li-Jia Qu

Tel/Fax: +86-10-6275 3018; Fax: +86-10-6275 3339

E-mail: qulj@pku.edu.cn

Received 31 October 2006; revised 12 January 2007; accepted 8 February 2007; published online 8 May 2007

Abbreviations: GA (Gibberellic acid); ABA (Abscisic acid); PDS (phytoene desaturase); CLA1 (1-deoxyxylulose 5-phosphate synthase); DXR (1-deoxyxylulose 5-phosphate reductoisomerase); HMGR (3-hydroxy-3-methylglutaryl coenzyme A reductase); IPI (isopentenyl pyrophosphate: dimethyllallyl pyrophosphate isomerase); GGPS (geranylgeranyl pyrophosphate synthase); CHS (chalcone synthase); GGRS (geranylgeranyl reductase); CPS (copalyl diphosphate synthase); KS (ent-Kaurene synthase); PSY (phytoene synthase); ZDS (z-carotene desaturase); LYC (lycopene cyclase) hydrocarbon compounds formed through the condensation of isoprenoids [1]. They play an important role in a large number of physiological processes in plants. Carotenoids act as accessory pigments in photosynthesis and form the basic structural units of photosynthetic antennae. They also serve as photo-protection agents by quenching singlet oxygen that might otherwise damage chlorophyll. Moreover, carotenoids serve as precursors in the biosynthesis of vitamin A and abscisic acid (ABA). Many clinical studies have demonstrated that carotenoids function as free radical quenchers in many physiological pathways [2].

Although carotenoids are synthesized in plastids, all the enzymes involved in their biosynthesis are encoded by nuclear genes. As shown in Figure 1, DMAPP is synthesized via the mevalonate (MVA) pathway or non-MVA pathway before being converted into geranylgeranyl 
pyrophosphate (GGPP) through a series of condensation reactions. GGPP is then shuttled into three routes: 1) to form $\beta$-carotene, which eventually leads to the synthesis of ABA [1, 3]; 2) to form gibberellins (GAs) [4] and 3) to form chlorophyll [5]. Phytoene desaturation is an important step in the $\beta$-carotene biosynthesis pathway. The enzyme responsible for this step is phytoene desaturase (PDS), the

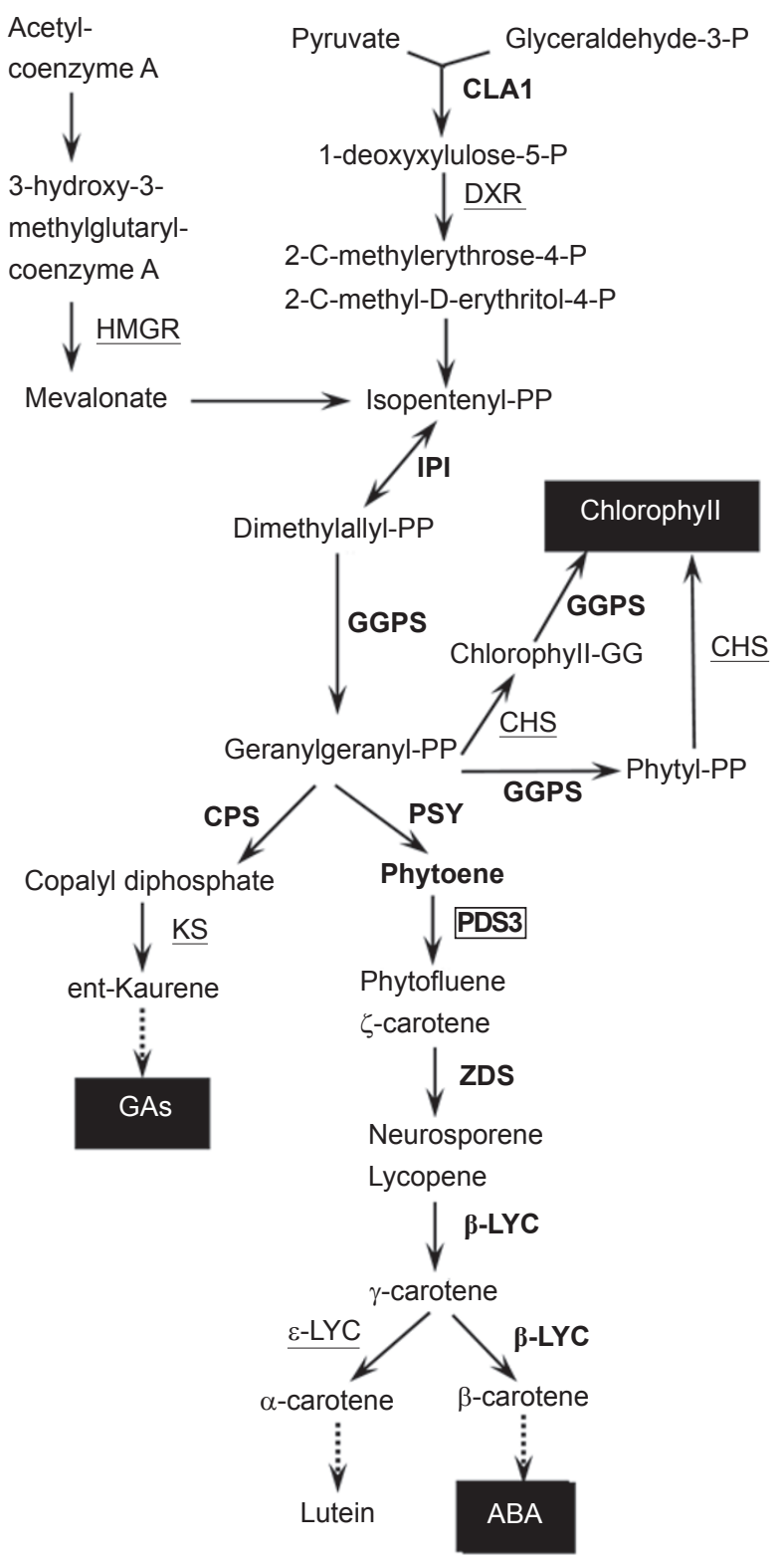

Figure 1 Carotenoid, chlorophyll and GA biosynthesis pathways. The enzymes for which the expression of their corresponding coding genes was quantitatively analyzed by RT-PCR in this paper are boldfaced, and the other enzymes are underlined. PDS3 is boldfaced and bracketed. target of a commercially important herbicide Norflurazon [6]. The $P D S$ gene was first cloned from cyanobacterium [7]. Homologous $P D S$ genes were then cloned from soybean [8], pepper [9], tomato [10,11], Arabidopsis [12] and maize [13]. These homologous proteins share about $60 \%$ identity and $75 \%$ similarity to the cyanobacterium PDS in amino acid sequences.

The phytoene desaturation step in carotenoid biosynthesis has also been studied through the use of mutants in Arabidopsis. Genetic screen for phytoene desaturation defect resulted in the isolation of two related mutants, $p d s 1$ and $p d s 2$, both defective in phytoene desaturation and exhibiting the albino phenotype $[14,15]$. The fact that the PDS1 and $P D S 2$ genes encode 4-hydroxyphenylpyrurate dioxygenase and prenylphytyltransferase respectively [14, 15], suggests that plastoquinone is a critical intermediate electron carrier for electron transport in the phytoene desaturation reaction. Another Arabidopsis mutant, immutans, accumulates phytoene in variegated white tissues. The IMMUTANS gene encodes plastid terminal oxidase, the disruption of which prevents oxidation during chlorophyll biosynthesis and results in the accumulation of phytoene [16, 17]. However, mutations in the gene encoding the phytoene desaturase itself (designated PDS3) [14] have not been reported. Expression analysis of the gene (PDS3) encoding the phytoene desaturase indicated that it is highly regulated by light $[18,19]$ in tomato. Nevertheless, analysis of the immutans mutant showed that the expression of PDS3 gene was not necessarily correlated to the amount of pigments in the cells [20].

We have obtained a new recessive albino mutant from a collection of T-DNA insertion mutants of Arabidopsis generated in our center. Molecular evidence demonstrated that the mutant phenotype resulted from a T-DNA insertion within the PDS3 gene. Further analysis showed that the mutant accumulated phytoene, and exogenous $\mathrm{GA}_{3}$ could partially rescue the dwarf phenotype of the mutant. Molecular genetic and the microarray techniques were used to investigate the role of phytoene desaturation and the underlying causes of albino and dwarf phenotypes in the $p d s 3$ mutant.

\section{Material and Methods}

\section{Mutagenesis and growth of Arabidopsis}

Arabidopsis of Columbia ecotype (col-0) were grown at $22{ }^{\circ} \mathrm{C}$ under long-day conditions. The floral dip method [21] was used to generate transgenic Arabidopsis with Agrobacterium GV3101 harboring pSKI015 [22]. Harvested seeds were screened on 1/2 MS medium containing $10 \mu \mathrm{g} / \mathrm{mL}$ of PPT (DL-Phosphinothricin). Green seedlings were transferred to soil and placed under the same conditions as described above. For determination of GAs, as well as for RT-PCR, HPLC microarray, and microscope analyses, the seedlings were transferred onto $1 / 2 \mathrm{MS}$ medium in flask instead of soil. 


\section{Identifying the flanking sequences of the insertions}

Genomic DNA was extracted from Arabidopsis leaves by the CTAB method [23] and served as the template for TAIL-PCR [24] in the amplification of flanking regions. The DNA fragments amplified by TAIL-PCR were recovered from agarose gels and purified before sequencing using an ABI 377 automated DNA sequencer (Applied Biosystems, USA). The output sequences were then searched against the Arabidopsis genome sequence database (GenBank) using BLAST to localize the position of the T-DNA insertion as described previously [25]
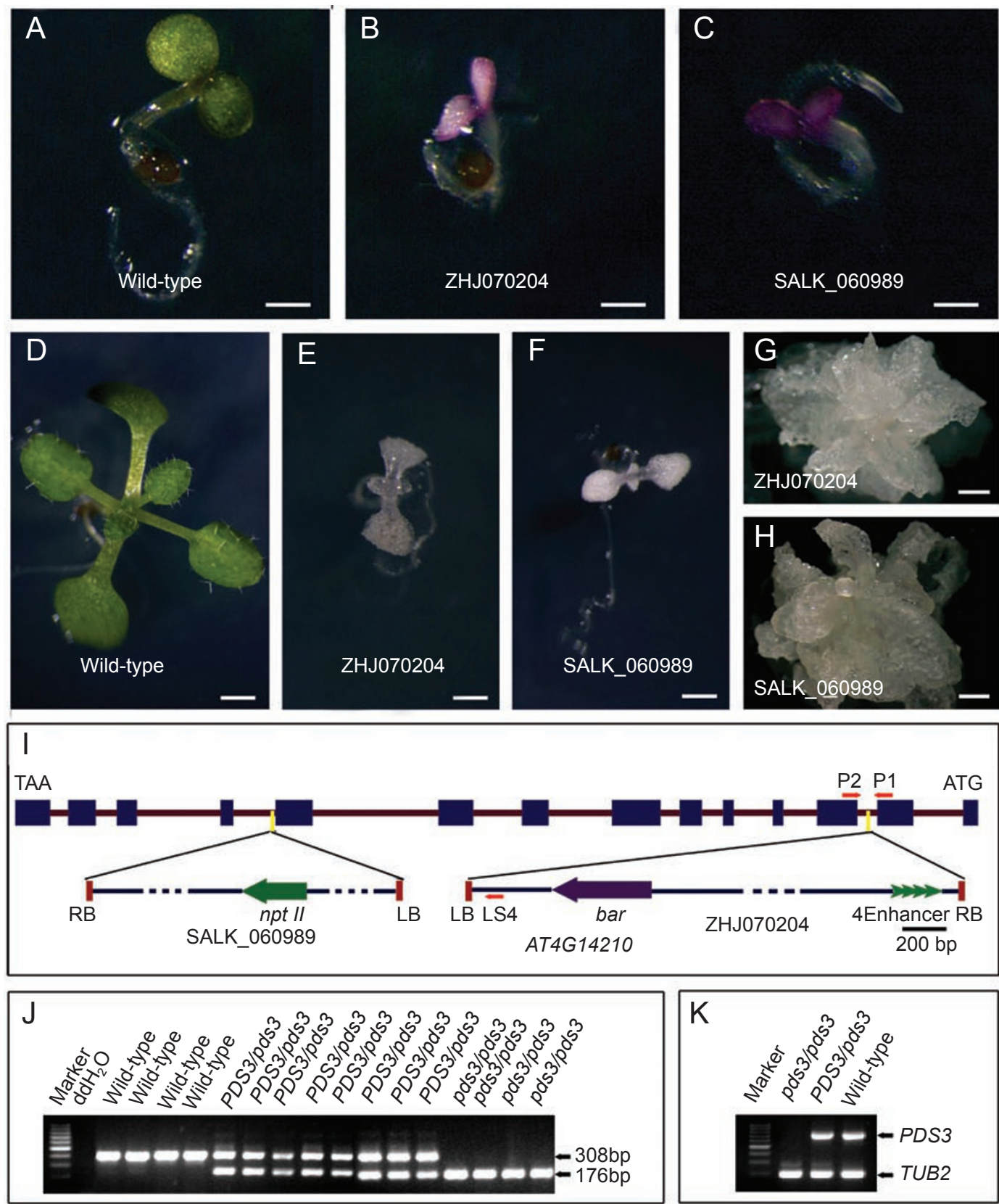

Figure 2 Phenotypes of the $p d s 3$ mutant. After cold treatment at $4{ }^{\circ} \mathrm{C}$ for three days, wild-type and the $p d s 3$ mutant seeds were grown at $22 \pm 2{ }^{\circ} \mathrm{C}$ under long-light conditions and compared at different developmental stages. (A) 5-day-old wild-type. (B) 5-day-old ZHJ070204. (C) 5-day-old SALK_060689. (D) 21-day-old wild-type. (E) 21-day-old ZHJ070204. (F) 21-day-old SALK_060689. (G) 42-day-old ZHJ070204. (H) 42-day-old SALK_060689. (I) Schematic representation of T-DNA insertion sites in the mutants of ZHJ070204 and SALK_060689. (J) Co-segregation analysis of the pds3 mutant. (K) Expression analysis of the PDS3 gene in wild-type and the $p d s 3$ mutant. The $\beta$-tubulin gene $T U B 2$ was used as an internal control. Scale bar $=1 \mathrm{~mm}$. 


\section{Co-segregation analysis}

The primers LS4, P1 and P2 were designed to check the T-DNA insertion in the $p d s 3$ mutant (Figure 2I). The primer sequences used were: LS4: 5'-TTG GTA ATT ACT CTT TCT TTT CCT CC-3'; P1: 5'-AGA GGA CGA CAT GGT TCA CAG T-3'; P2: 5'-CGG TCA ATG CTG GAG CTA GTA T-3'. Five pmol of each of the three primers and $20 \mathrm{ng}$ of genomic DNA were present in $25 \mu \mathrm{L}$ of the PCR reaction mix. Following denaturation at $95^{\circ} \mathrm{C}$ for $2 \mathrm{~min}$, the reaction was then subjected to 30 cycles of denaturation $\left(94{ }^{\circ} \mathrm{C}\right.$ for $\left.30 \mathrm{sec}\right)$, annealing $\left(58^{\circ} \mathrm{C}\right.$ for $\left.30 \mathrm{sec}\right)$ and extension $\left(72^{\circ} \mathrm{C}\right.$ for $\left.45 \mathrm{sec}\right)$. PCR products were then analyzed on $2 \%$ agarose gels.

\section{Cloning of the Arabidopsis PDS3 gene}

Two primers were designed based on the available cDNA sequence (Accession No: AF360196) to amplify the Arabidopsis PDS3 gene, 5'-ATG GTT GTG TTT GGG AAT GTT TCT GCG GCG A-3' and 5'-TCA TGA TGA TGA TAC TGT TGC CTC CGA CAA CTT-3'. Using $10 \mathrm{ng}$ of first-strand cDNA from wild-type Arabidopsis leaves as the template, PCR was performed in a $50-\mu \mathrm{L}$ volume with $20 \mathrm{pmol}$ of each primer and high-fidelity $P f u$ DNA polymerase. Following a one-minute initial denaturation step at $94{ }^{\circ} \mathrm{C}$, the amplification went through 30 cycles consisting of denaturation at $94{ }^{\circ} \mathrm{C}$ for 30 sec, annealing at $65^{\circ} \mathrm{C}$ for $30 \mathrm{sec}$, followed by extension at $72{ }^{\circ} \mathrm{C}$ for $2 \mathrm{~min}$. The amplified product was recovered from low-melting point agarose gel and then ligated into the EcoR V site of pBS after purification. Recombinant plasmids containing the $P D S 3$ gene in both sense (designated as pBPDS3) and anti-sense orientations (designated as pAPDS3) were identified through sequencing.

\section{Arabidopsis transformation}

After being treated with the appropriate restriction enzymes, the insert from recombinant plasmid pBPDS was recovered from lowmelting point agarose gels, purified and then ligated into the plant expression vector pWM101, to generate pWPDS3 (Figure 3A). PDS3$p d s 3$ heterozygous mutant plants were used as the recipients for Agrobacterium-mediated transformation by the floral dip method. The seeds of transgenic plants were screened on 1/2 MS medium containing $25 \mu \mathrm{g} / \mathrm{ml}$ of hygromycin and the surviving seedlings were transferred into soil after a one-week recovery period on nonselective MS medium.

Two primers were designed based on the HPTII gene sequence in pWM101 and used to confirm the success of the transformation. The primer sequences were: $5^{\prime}$-AGG AAT CGG TCAATA CAC TAC A-3' and 5'-ACT ATC GGC GAG TAC TTC TAC A-3'. PCR was performed in $25 \mu \mathrm{L}$ volumes with 5 pmol of each primer and $10 \mathrm{ng}$ of genomic DNA. Following a two-minute initial denaturation step at $95{ }^{\circ} \mathrm{C}$, the amplification proceeded through 30 cycles consisting of denaturation at $94{ }^{\circ} \mathrm{C}$ for $30 \mathrm{sec}$, annealing at $58^{\circ} \mathrm{C}$ for $30 \mathrm{sec}$, followed by extension at $72^{\circ} \mathrm{C}$ for $1 \mathrm{~min}$. PCR products were analyzed on $1 \%$ agarose gels.

\section{Quantitative RT-PCR analysis}

Total RNA was extracted from the leaves of 4-week-old plants (WT, PDS3-pds3, pds3-pds3 mutants) using Qiagen RNAeasy Plant Mini Prep kits (Qiagen, USA). First-strand cDNA synthesis was performed in a 20 - $\mu \mathrm{L}$-reaction volume consisting of $3 \mu \mathrm{g}$ of total RNA, oligo$\mathrm{dT}_{12-18}$ and SuperScript ${ }^{\mathrm{TM}}$ reverse transcriptase (GIBCO BRL). The reaction was allowed to proceed at $42{ }^{\circ} \mathrm{C}$ for $50 \mathrm{~min}$ before being terminated by treatment at $70{ }^{\circ} \mathrm{C}$ for $15 \mathrm{~min}$. Quantitative RT-PCR
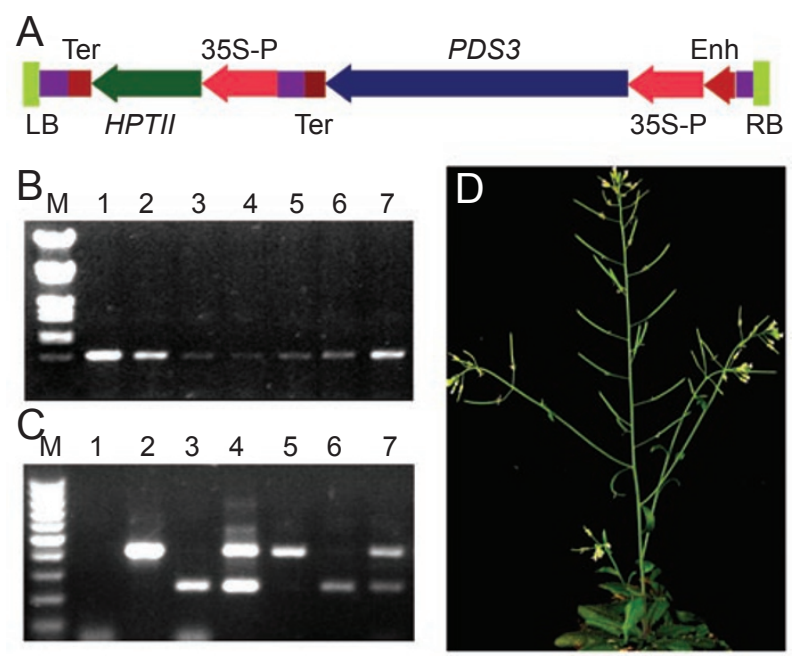

Figure 3 Phenotype rescue of the $p d s 3$ mutant by a wild type $P D S 3$ transgene. (A) The pWPDS3 construct. Different elements of the vector are represented by boxes with different colours; the transcriptional orientation is indicated by arrow. LB, the left border of T-DNA; RB, the right border of T-DNA; Ter, CaMV 35S ployA; 35S-P, CaMV $35 \mathrm{~S}$ promoter; HPTII, hygromycin phosphotransferase gene; $P D S 3$, wild-type phytoene desaturase gene; Enh, CaMV 35S enhancer. (B) PCR amplification of HPT II genes. M: $\lambda / E c o \mathrm{R}$ I + Hind III marker; 1-7: seven independent plants transformed with pWPDS3. The 568-bp band is amplified from HPT II genes. (C) Genotype characterization of the three transgenic plants using the three primers LS4, P1 and P2. M: 100-bp ladder; 1: blank control; 2: wild-type; 3: the $p d s 3-p d s 3$ homozygous mutant; 4: PDS3-pds3 heterozygous mutant; 5-7: three independent plants of different genotypes were segregated from heterozygous $P D S 3-p d s 3$ mutant transformed with pWPDS3. (D) The phenotype of $p d s 3-p d s 3$ homozygous plant was rescued by transforming with pWPDS3.

analysis was performed using the following primers: TUB2 ( $\beta$-tubulin): 5'-GTT CTC GAT GTT GTT CGT AAG-3' and 5'-TGT AAG GCT CAA CCA CAG TAT-3'; CPS: 5'-AAG TGT CCG CAG ATG TAT TC-3' and 5'-ATA TAG TTG CAG CCG CTA AG-3'; PDS3: 5'-TTG CAG TGG AAG GAA CAC TC-3' and 5'-ACT CTT AAC CGT GCC ATC GT-3'; CLA1: 5'-GAG TAT GCT CGA GGA ATG ATA A-3' and 5'-AGA CGA ATA GAT TGC ACA GAA G-3'; IPI: 5'-ATG CTG TTC AGA GAC GAC TCA T-3' and 5'-TCA CCA GCT CCT TAA GCT CTT C-3'; GGPS: 5'-ATC CAC GAA GCG ATG CGT TAC T- $3^{\prime}$ and 5'-CAC TTC CTC CAC CAA CAA TAG C-3'; PSY: 5'-GAA CCG AAG TAG AAG AAT TG-3' and 5'-GAT CAT CGA AGT TCT GGT AT-3'; ZDS: 5'-AGA TAG AGG TGG CAG AAT CC-3' and 5'-GGT GTT AGA ACG CAC TGA AG-3'; LYC: 5'-TGG TCT GGT GCT GTT GTC TAT G-3' and 5'-GCT CGT CTT CCT CAA TCC TCT T-3'; GGRS: 5'-CAT CGA GCG TAA GAT GGA CAA T-3' and 5'-GCG ACA CAT CAT CTC CAA CAT A-3'; PDS1: 5'-AAG TAG AAG ACG CAG AGT CA-3' and 5'-ATC AGA GCC AGA TGT TGT AG-3'; IM(immutans): 5'-CCT TAC AGA CTC GGT TAT TA-3' and 5'-TTA CTG GTC TTC GAG TAT TG-3'.

RT-PCR was performed in a $25 \mu \mathrm{L}$ reaction volume consisting 
of $0.25 \mu \mathrm{L}$ of the first-strand cDNA product and $5 \mathrm{pmol}$ each primer pairs, with one pair for a specific gene and the other for the tubulin gene (internal control). PCR mixes were subjected to 24 cycles (with the exception of 30 cycles for the CPS gene and 28 cycles for the $P D S 1$ and $I M$ genes). Following a one-minute initial denaturation step at $94{ }^{\circ} \mathrm{C}$, the amplification consisted of denaturation at $94{ }^{\circ} \mathrm{C}$ for $30 \mathrm{sec}$, annealing at $54{ }^{\circ} \mathrm{C}$ for $30 \mathrm{sec}$, followed by extension at $72{ }^{\circ} \mathrm{C}$ for $1 \mathrm{~min}$. The products were analyzed on $1.5 \%$ agarose gels and the relative intensity of each band was determined and compared. For each gene analyzed, RT-PCR was repeated independently for three times, the average relative intensity counts were calculated and $t$-tests for pairwise comparisons of means were carried out.

\section{HPLC analysis of carotenoids}

One-month-old wild-type Arabidopsis seedlings were transferred onto MS medium containing $30 \mu \mathrm{g} / \mathrm{mL}$ of Norflurazon and allowed to grow for two weeks before being collected for carotenoid extraction. Meanwhile, six-week-old wild-type and the $p d s 3$ mutant plants were also collected for carotenoid extraction. The protocol used for the extraction of lipid-soluble carotenoids was slightly modified from the previous report [14]. Carotenoids were separated by reverse-phase HPLC on a ZOR BAX 300SB C18 column (4.6 $\mathrm{mm}$ ID $\times 250 \mathrm{~mm}$, Gilson, France) using a 45 -min gradient of ethyl acetate $(0$ to $100 \%)$ in acetonitrile-water $(9: 1)$ at a flow rate of $1 \mathrm{~mL}$ per min. Retention times relative to known standards, using detection at both 296 and $440 \mathrm{~nm}$, were used to identify the chlorophylls and carotenoids. Since no phytoene standard was available in this laboratory, carotenoids extracted from wild-type Arabidopsis treated with the herbicide Norflurazon, in which phytoene was accumulated [26], were used as standards.

\section{Microarray analysis}

Microarray experiments and data analysis were performed according to a previously established protocol [27]. Briefly, $100 \mu \mathrm{g}$ of total RNA from the $p d s 3$ mutants and wild-type seedlings was used to synthesize $\mathrm{Cy} 3$ - and $\mathrm{Cy} 5$-labeled cDNA, respectively. A combination of wild-type cDNA and an equal amount of mutant cDNA was used to simultaneously probe a glass slide containing duplicate arrays in three microarray experiments. Each experiment was repeated with reverse labeling.

\section{Electron microscope analysis}

Sections of leaf tissue were prepared for EM observation according to a previously described protocol [28] with minor modifications. Briefly, Arabidopsis leaf tissue was fixed with glutaraldehyde, then fixed with osmium tetroxide and dehydrated in an ethanol series before being infiltrated with Spurr (Dow Chemical Co., USA). Polymerization was conducted at $70^{\circ} \mathrm{C}$ for $8 \mathrm{~h}$. Specimens were sliced to yield ultra-thin sections (LKB-8800, Sweden) and then stained with uranyl acetate and alkaline lead citrate before being examined with a JEM-100S transmission electron microscope.

\section{Results}

\section{Isolation of a new albino mutant}

From a collection of T-DNA insertion mutants of Arabidopsis that we recently generated, $\mathrm{T}_{2}$ seeds of 2500 lines were screened for observable phenotypes. One line (ZHJ070204) with an albino phenotype was further analyzed. The homozygous mutant was completely albino and unable to grow in soil. However, it could survive and grow on $1 / 2$ MS medium containing 1\% sugar. While germinating normally, the mutant had purple cotyledons that were gradually bleached to complete white with extended growth period (Figure 2B, 2E, 2G). The mutant was greatly retarded in development, displaying a severe dwarf phenotype with small rosette leaves. No bolting was observed in any of these cultured mutant plants (Figure 2G).

The mutant phenotype co-segregates with the T-DNA insertion

As a first step in revealing the molecular nature of the mutation, the flanking sequences of the T-DNA insertion site were TAIL-PCR-amplified and sequenced. One insertion was located in the second intron of the phytoene desaturase gene (At4g14210) in this mutant (Figure 2I). We designated this mutant as $p d s 3$ for reasons discussed in the following sections. When T3 seeds from different T2 green lines were planted on MS medium without PPT selection, the $\chi^{2}$ test showed that the number of green plantlets vs. that of albino was $3: 1$, suggesting that the phenotype was caused by a single recessive mutation.

To check whether the T-DNA insertion co-segregates with the albino phenotype, DNA from T3 progenies were used as templates for PCR analysis (Figure 2J). Primers were designed to amplify a 308-bp fragment from wild-type plant and a 176bp fragment from the homozygous mutant. In all heterozygous mutants, both fragments were amplified, indicating that the T-DNA insertion in the $P D S 3$ gene co-segregated with the albino phenotype (Figure 2J). SALK_060989, a SALK T-DNA line with a T-DNA insertion in the tenth intron of At4g14210 (Figure 2I) from the Arabidopsis Biological Resource Center [29], displayed the similar phenotypes with ZHJ070204 (Figure 2C, 2F, 2H). These data further support that the albino phenotype was caused by the T-DNA insertion. This conclusion is also consistent with the fact that the PDS3 transcript was absent in the albino mutant as shown by RT-PCR analysis (Figure $2 \mathrm{~K}$ ).

\section{The PDS3 gene complements the albino and dwarf phe- notypes}

To find out if the disruption of $P D S 3$ was responsible for the albino phenotype, we attempted to rescue the phenotype by introducing a cDNA of the PDS3 gene driven by the $35 \mathrm{~S}$ promoter back into the mutant. When the vector harboring the wild type $P D S 3$ gene was constructed (Figure 3A) and transformed into heterozygous $p d s 3$ plants, we were able to obtain progeny homozygous for $p d s 3$ yet with the wild type phenotype (Figure 3B-D). These transgenic plants homozygous for $p d s 3$ carried a new PDS3 transgene (Figure 3C). 


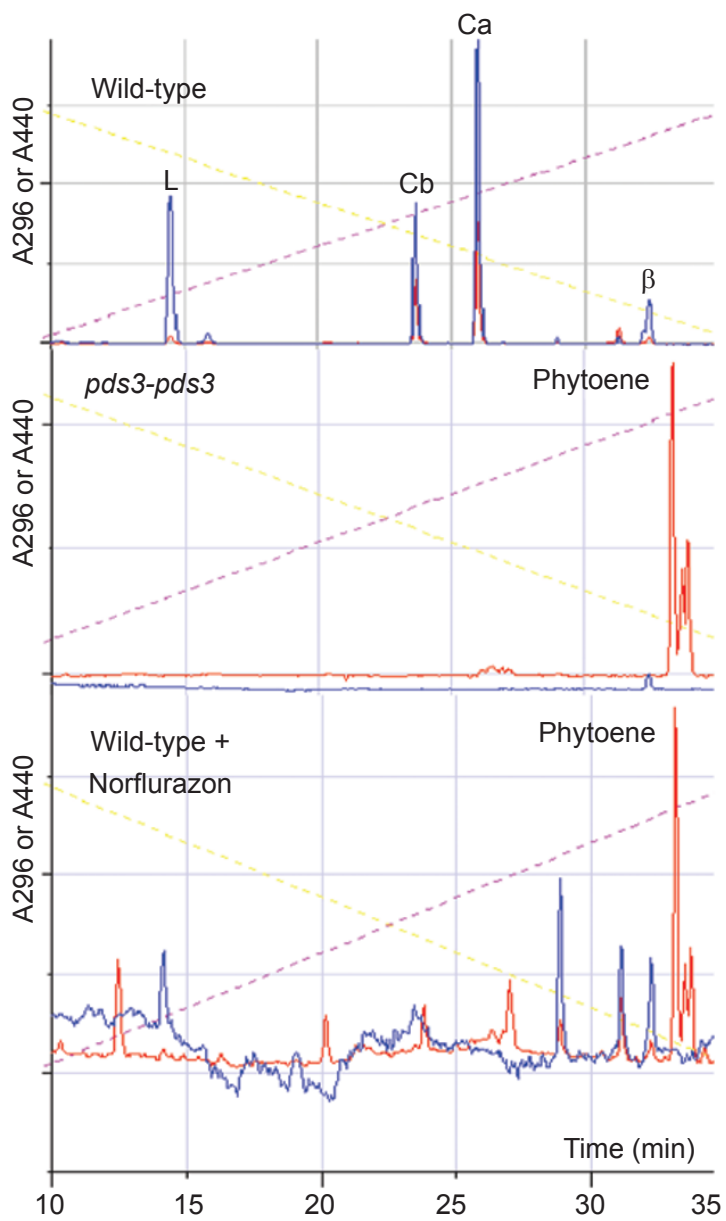

Figure 4 HPLC analysis of carotenoids in leaves from wild-type, $p d s 3$ mutant and Norflurazon-treated wild-type plants. The absorbance spectra at $296 \mathrm{~nm}$ and $440 \mathrm{~nm}$ are indicated by red and blue lines, respectively. $\mathrm{L}$, lutein; $\mathrm{Cb}$, chlorophyll $\mathrm{b}$; $\mathrm{Ca}$, chlorophyll a; $\beta, \beta$-carotene.

Further phenotype analysis of the rescued plants indicated that the PDS3 gene could fully complement the albino phenotype and all other associated abnormalities, including dwarfism, lethality, and infertility (Figure 3D). To further confirm the contribution of the $P D S 3$ gene disruption to the observed phenotype of the $p d s 3$ mutant, an RNAi transgene for $P D S 3$ under the strong constitutive $35 \mathrm{~S}$ promoter was constructed and introduced into wild type Arabidopsis. All the RNAi transgenic plants examined exhibited, to different extent, similar phenotypes to that of the $p d s 3$ mutant (Supplemental information, Figure S1). This result further confirmed that the phenotype of our initial $p d s 3$ mutant was strictly due to the disruption of $P D S 3$ gene, thus providing the basis for designation of the mutant as $p d s 3$.
High levels of phytoene accumulate in the pds 3 mutant

HPLC was performed to examine carotenoid and other pigment contents in the $p d s 3$ mutant. The analysis showed that the chlorophyll and $\beta$-carotene peaks detected in the wild type plants were absent in the $p d s 3$ mutant (Figure 4). Furthermore, the three main peaks that were observed in the $p d s 3$ mutant occurred at the same position where phytoene would normally appear in plants treated with the herbicide Norflurazon (Figure 4). The three peaks are probably the cis- and trans-isoforms of phytoene. These data indicated that phytoene accumulated in the $p d s 3$ mutant due to the loss of phytoene desaturation activity, which also led to the absence of chlorophyll and $\beta$-carotene.

\section{Dwarf phenotype of pds 3 might result from GA defi- ciency}

The finding that the $p d s 3$ mutant was severely dwarfed, together with the fact that GGPP is required for the biosynthesis of carotenoid, chlorophyll and GAs, raised the possibility that GA biosynthesis in the $p d s 3$ mutant was disrupted. Semi-quantitative RT-PCR analysis showed that expression of the gene coding for copalyl diphosphate synthase (CPS), the first enzyme in the committed GA biosynthesis pathway, was largely reduced in the homozygous
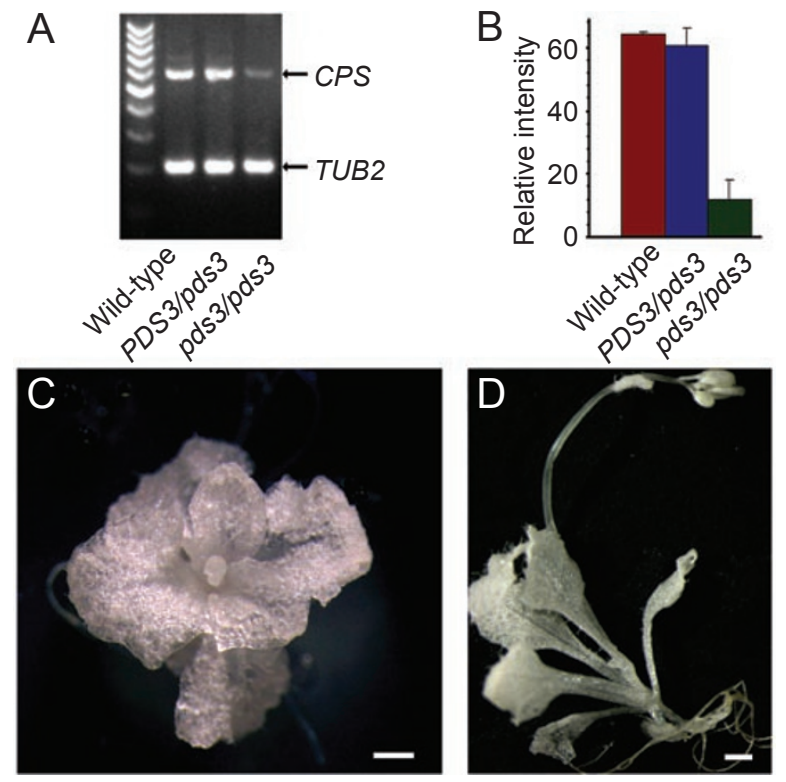

Figure 5 The $p d s 3$ mutant might be deficient of GAs. (A, B) Semiquantitative analysis of CPS gene expression by RT-PCR. M: 100-bp ladder; TUB2: $\beta$-tubulin gene as the internal control. (C) 35-day-old $p d s 3$ seedling grown on $1 / 2 \mathrm{MS}$ medium. (D) 35 -day-old $p d s 3$ seedling transferred to $1 / 2 \mathrm{MS}$ medium containing $1 \mu \mathrm{g} / \mathrm{mL}$ of exogenous $\mathrm{GA}_{3}$ after growing on $1 / 2 \mathrm{MS}$ for two weeks. Scale bar $=1 \mathrm{~mm}$. 
$p d s 3$ mutant but not affected in the wild-type or heterozygous $p d s 3$ plants (Figure 5A and 5B). This suggested that the $p d s 3$ mutation affected the expression of genes involved in commitment to GA biosynthesis. We then tested whether exogenous GA could rescue the dwarf phenotype of the $p d s 3$ mutant. We observed that after transferring the $p d s 3$ mutant plants to $1 / 2 \mathrm{MS}$ plate containing $1 \mu \mathrm{g} / \mathrm{mL}$ of exogenous $\mathrm{GA}_{3}$, these plants grew bigger, exhibited elongated petioles and normal bolting, and were capable of setting flowers in contrast to those grown on the $1 / 2 \mathrm{MS}$ without $\mathrm{GA}_{3}$ (Figure 5C and 5D). In comparison, the $p d s 3$ plants without exogenous GAs were infertile with degenerate stamens and pistils (data not shown). Taken together, these results indicate that the dwarf phenotype in the $p d s 3$ mutant might be caused by the deficiency of GAs.

Disrupting PDS3 gene results in inhibition of many other genes involved in carotenoid, chlorophyll, and GA biosynthesis pathways

To systematically examine possible effects of $p d s 3$ mutation on other related biosynthetic pathways, nine representative genes were chosen for semi-quantitative RT-PCR analysis (shown in Figure 1). These genes include $C L A 1$ [30], IPI, GGPS, PSY, ZDS and $L Y C$ that are directly involved in the carotenoid biosynthesis pathway, GGRS that is required in the side-branched chlorophyll biosynthesis, and $P D S 1$ and $I M$ that are functionally related to phytoene desaturation. The results showed that the nine genes shared a similar pattern of expression. The expression level of most genes was comparable between wild-type and heterozygous $p d s 3$ plants but clearly reduced in the homozygous $p d s 3$ mutant plants (Figure 6). For example, the expression of GGPS and $P S Y$ in the $p d s 3$ mutant was significantly lower than that in the wild-type plant. These data indicated that the expression of many genes involved in the carotenoid, GA, and chlorophyll biosynthesis pathways was lowered by disruption of the $P D S 3$ gene.

\section{Metabolic pathways and nuclear encoded chloroplastic genes were affected in the pds 3 mutant}

To further investigate effects of the mutated PDS3 gene on other metabolic pathways, we compared the genome expression profile of the $p d s 3$ mutant seedlings with that of the wild type using microarray analysis. The microarray contained a total of 9216 ESTs representing approximately 6120 unique genes [27]. A total of 596 unique genes exhibited twofold or more differential expression in $p d s 3$ in comparison with wild-type, with 330 being up-regulated and 266 down-regulated. Of the 596 differentially regulated genes, 392 have known functions or share sequence similarity with genes of known functions, whereas the rest 204 were genes with unknown function (Supplemental information, Table S1). Of the 392 genes with known or predicted functions, 126 or about $21 \%$ of them can be clas-

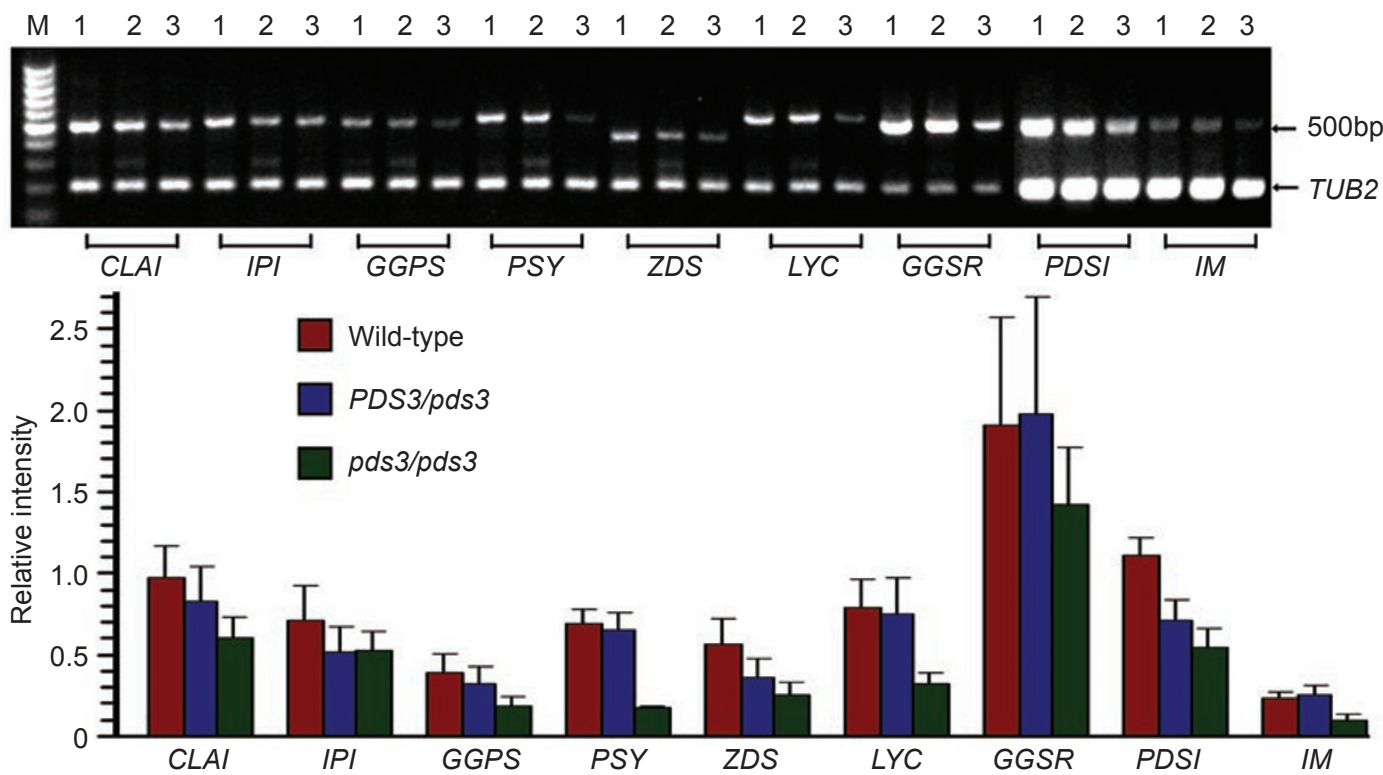

Figure 6 Expression analysis of genes involved in related biosynthetic pathways in wild-type (1), PDS3-pds3 heterozygous mutant (2), and $p d s 3$-pds 3 homozygous mutant (3). M: 100-bp ladder; TUB2: $\beta$-tubulin gene as the internal control. Primers designed for IPI gene can be used to amplify both AtIPI1 and AtIPI2 transcripts. 
Table 1 Number of genes involved in different functional groups up-regulated or down-regulated by at least two-fold in the $p d s 3$ mutant

Pathways

\section{Down-regulated}

Calvin cycle

Photosynthetic light reactions

Glycolysis

Trichloroacetic acid cycle

Cell wall synthesis

Biosynthesis of terpenoid or chlorophylls

Phenylpropanoid biosynthesis

Protein synthesis in chloroplast

Protein synthesis in cytoplasm

Ubiquitin - proteasome pathway

Amino acid synthesis pathways

Transcription factors

\section{Up-regulated}

Cell wall degradation and cell wall proteins

Ethylene biosynthsis and ethylene responsive genes

Stress tolerance

Protein synthesis in cytoplasm

Nitrogen assimilation

Branched-chain amino acid catabolism

Sulfur assimilation

Transcription factors
Rubisco small subunit 1b, 2b, 3b, enolase, 2 glyceraldehyde 3-phosphate dehydrogenase, fructose-bisphosphate aldolase, nucleotide sugar epimerase, phosphoribulokinase

127 chlorophyll a / b binding proteins, photosystem I reaction center subunit II, IV, V, 2 proteins involved in electron transport

$6 \quad \beta$-amylase, alcohol dehydrogenase, lactate dehydrogenase, enolase, fructose-bisphosphate aldolase, phosphorglyerate mutase

3 Citrate synthase, pyruvate dehydrogenase, isocitrate dehydrogenase

43 glucosyltransferase, UDP-glucose:glycoprotein glucosyltransferase

5 Mevalonate diphosphate decarboxylase, CLA1 protein, geranylgeranyl reductase, protoporphyrinogen oxidase, lupeol synthase protein

5 Glutathione-conjugate transporter, 2 GSTs, 2 cytochrome P450

32 chloroplast $50 \mathrm{~S}$ ribosomal protein, chloroplast initiation factor

13560 S ribosomal proteins, 3 other ribosomal proteins, arginyl-tRNA synthetase, 2 translation initiation factor, 2 RNA helicases

2 ubiquitin-conjugating enzyme E2, 26S proteasome subunit, 26S proteasome regulatory subunit Glycine dehydrogenase, Proline synthetase, phosphoribosylglycinamide synthetase, $\Delta-1$ pyrroline 5-carboxylase synthetase

10 CCR4-associated factor, MADS box transcription factors, DNA binding protein CCA1, transcriptional adaptor, myb-related protein, ring-box protein, bHLH transcription factor, MYB-related transcription factor, bZIP transcription factor TGA1, basic leucine zipper transcription activator

12 Xyloglucan endo-1,4- $\beta$-D-glucanase, pectinacetylesterase, pectinesterase, XET, 2 xylosidases, $\beta$-galactosidase, 2 peroxidases, 2 glycine-rich proteins, proline-rich protein

ACC synthase, ACC oxidase, ethylene responsive element binding factor, ethylene-respon sive protein

Drought-induced protein, wound-responsive protein, water stress-induced protein, saltinduced protein, aluminum-induced protein, harpin-induced protein, nematode-resistance protein, cold-acclimation protein

56 S ribosomal protein, $40 \mathrm{~S}$ ribosomal protein, other ribosomal protein, chaperonin, translation initiation factor

3 Glutamate dehydrogenase, glutamine synthetase, cysteine synthase

53 branched-chain alpha keto-acid dehydrogenases, branched-chain amino acid aminotransferase, aminotransferase

42 GSTs, sulfate transporter, cysteine synthase

$7 \mathrm{C} 2 \mathrm{H} 2$ zinc finger transcription factor, transcription regulator protein, 2 DNA-binding proteins, transcriptional co-repressor SIN3A, transcription factor-like protein light-induced protein, zinc-finger protein Lsd1

${ }^{\mathrm{a}}$ Rubisco, ribulose-1,5-bisphosphate carboxylase/oxygenase; GST, glutathione S-transferase; XET, xyloglucan endotransglycosylase; ACC, 1-aminocyclopropane-1-carboxylic acid.

sified into 20 different metabolic pathways, 12 of which are repressed while 8 are enhanced (Table 1), according to criteria previously described [27].
After searching against MAtDB [31], 93 out of the 596 differentially-expressed genes were found to code for products that are targeted to chloroplast, with 34 be- 
ing up-regulated and 59 down-regulated (Supplemental information, Table S2). Among these nuclear encoded chloroplastic genes, seven chlorophyll-binding protein genes and three photosystem protein genes, involved in the photosynthesis and the Calvin cycle, were down-regulated, indicating that both light and dark reactions were repressed (Table 1). These data are consistent with the notion that lack of carotenoids and chlorophyll in the albino $p d s 3 \mathrm{mu}-$ tant would result in the repression of chlorophyll binding protein genes and photosynthesis. Further, the expression level of $C L A 1$ and GGRS from the microarray analysis was also significantly lower in the $p d s 3$ mutant than that in the wild-type plant (Table 1), which is well corroborated with the RT-PCR analysis shown above (Figure 6).

\section{Chloroplast development is arrested at the proplastid stage in the pds 3 mutant}

To examine the development status of the plastids in the pds 3 mutant, mesophyll cell plastids from $5 \mathrm{~d}, 30 \mathrm{~d}$ and $90 \mathrm{~d}$ old $p d s 3$ mutant seedlings were analyzed by transmission electron microscopy. The data showed that in wild-type Arabidopsis chloroplasts and other plastids including the thylakoid membrane system developed normally in light (Figure 7A). In contrast, no mature plastids were found in the $p d s 3$ mutant. Only undifferentiated proplastids were found in the mesophyll cells at all three developmental stages examined, while no thylakoid membrane system
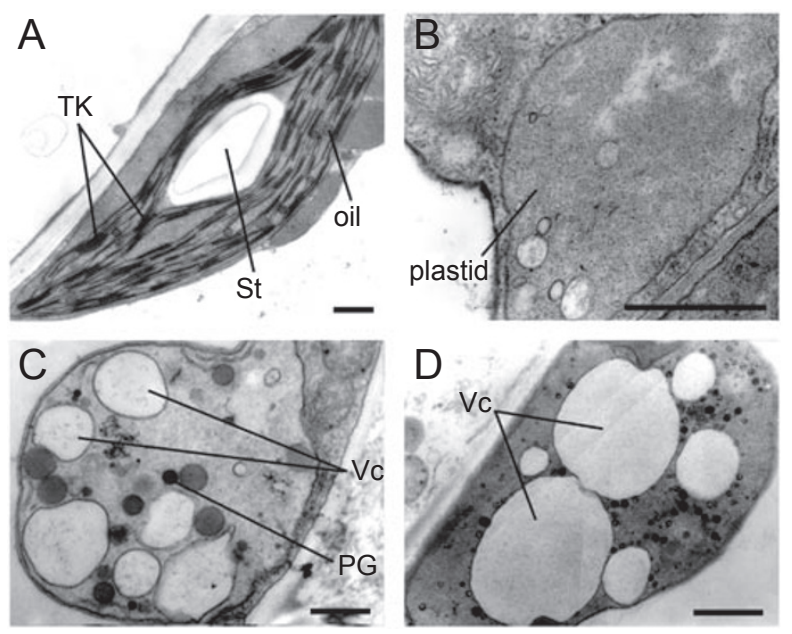

Figure 7 The effect of the $p d s 3$ mutation on plastid development. (A) wild-type. (B) Plastids in leaf mesophyll cells of purple seedlings 5 days after germination. (C) Plastids in leaf mesophyll cells of albino seedlings 30 days after germination. (D) Plastids in leaf mesophyll cells of albino seedlings 90 days after germination. St: starch granule; Tk: thylakoid; Oil: oil drops; PG: plastoglobule. Scale bar $=1.2 \mu \mathrm{m}$. formed in any of the cells examined (Figure 7C-D). Cells became increasingly vacuolated during development, which was also observed in another albino mutant apg2 [32]. These results indicated that proplastids in the mesophyll cells of the $p d s 3$ mutant were arrested at the undifferentiated stage and had lost the ability to develop into mature chloroplasts.

\section{DISCUSSION}

Disruption of the PDS3 gene resulted in failure of phytoene desaturation, albino and dwarf phenotypes in Arabidopsis

Carotenoid biosynthesis is an important metabolic pathway in Arabidopsis. The biosynthesis of chlorophylls, phytohormones, vitamins, and some important accessory pigments for photosynthesis such as $\beta$-carotene and xanthophylls all relates to the carotenoid biosynthesis pathway. Phytoene desaturase (PDS3) plays an important role in carotenoid biosynthesis. Two Arabidopsis PDS-related mutants, $p d s 1$ and $p d s 2$, with albino phenotypes, were found to accumulate high levels of phytoene, the substrate of PDS3, in their leaves $[14,15]$. However, mutations in PDS1 and $P D S 2$ genes only affected the availability of the electron carrier plastoquinone required for the phytoene desaturase function, but not the PDS3 enzyme itself. In our study, the albino mutant $p d s 3$ was obtained and analyzed. The $p d s 3$ mutant displayed albino and dwarf phenotypes with characteristics that included slow growth, small leaves, short petioles, absence of bolting and flowering, and infertility. Molecular and genetic evidence showed that an insertion of T-DNA into the PDS3 gene co-segregated with the mutant phenotype (Figure 2). Further analysis demonstrated that an intact copy of $P D S 3$ gene complemented the $p d s 3$ mutant phenotype (Figure 3 ) and that knock-down of PDS3 by RNAi in transgenic Arabidopsis resulted in variegated or albino, semi-dwarf or dwarf phenotypes (Supplemental information, Figure S1). Together with the evidence from the HPLC analysis showing that phytoene accumulated in the $p d s 3$ mutant (Figure 4), we concluded that the albino and dwarf phenotypes resulted from the disruption of the PDS3 gene.

The pds 3 mutation affects expression of about 1/10 of genes and causes multiple phenotypes

In order to investigate the molecular basis for the $p d s 3$ mutant phenotypes, microarray analysis of $p d s 3$ mutant seedlings was performed in comparison with wild-type. Our results indicated that expression of about $1 / 10$ of genes was either up- or down-regulated by two-fold or more in the pds 3 mutant (Supplemental information, Table S1). Among these differentially regulated genes, ninety-three encode chloroplast-destined proteins, with thirty-four 
genes being up-regulated and fifty-nine down-regulated (Supplemental information, Table S2). This significant change in gene expression for chloroplast proteins is consistent with the severely retarded development of the plastids (Figure 7), and with a previous report that nuclear encoded photosynthetic genes were repressed in bleached carotenoid-deficient mutant [33]. At least 26 pathways were found to be light-regulated in Arabidopsis [27]. In $p d s 3$, at least 20 metabolic pathways were affected. Interestingly, compared to the results of gene expression profiling in wild-type Arabidopsis, many light-regulated pathways were completely disrupted by the $p d s 3$ mutation. Due to the lack of chlorophylls, photosynthesis in $p d s 3$ was not functional; and photosynthesis-related pathways, such as photoreaction, photosystems, the Calvin cycle and chlorophyll biosynthesis, were all suppressed (Table 1 and Supplemental information, Table S2). Without photosynthesis, the mutant plants could only survive by taking carbons directly from the growth medium. Consequently, glycolysis and the Kreb's Cycle were suppressed while Nand S-assimilation related pathways were induced (Table 1 and Supplemental information, Table S2). Furthermore, the $p d s 3$ mutation resulted in a burst of induction of genes encoding stress-related proteins (Table 1 and Supplemental information, Table S2).

\section{Dwarfism might be caused by a deficiency of GAs}

The $p d s 3$ mutant showed a severe dwarf phenotype including retarded growth, short petioles, and absence of bolting (Figure 2G-2H). There are two potential explanations for the dwarfism. One hypothesis is that the dwarfism results from an energy deficiency due to the disruption of photosynthesis in the $p d s 3$ mutant; alternatively loss-offunction in $P D S 3$ gene may have impeded the biosynthesis of GAs, which is closely related to the $\beta$-carotene biosynthesis pathway (Figure 1). When exogenous GAs were added to the medium, the $p d s 3$ mutant, while still albino, showed normal bolting and extended petioles (Figure 5D). In addition, expression of the CPS gene, coding for the first enzyme in the committed GA biosynthesis pathway, was found to be down-regulated in the $p d s 3$ mutant, which probably led to a reduced production of GAs (Figure 5A and $5 \mathrm{~B}$ ). Therefore, the dwarfism of the $p d s 3$ mutant might be due to a deficiency of GAs.

\section{Possible mechanisms of gene expression changes in the pds 3 mutant}

In the $p d s 3$ mutant, not only the carotenoid pathway genes encoding downstream enzymes after phytoene desaturation, such as $Z D S$ and $L Y C$, were down-regulated, but also were the genes encoding upstream enzymes such as CLA1, IPI, GGPS and PSY, as well as those involved in the related GA and chlorophyll pathways, such as CPS and $G G R S$. Two possible mechanisms could be involved. First, photo-oxidative damage due to the lack of carotenoids may have inhibited the expression of not only photosynthetic genes but also other genes involved in carotenoid and GA biosynthesis pathways. It is reported that expression of nuclear-encoded photosynthetic genes could be inhibited through at least three signaling pathways based on the developmental status of the plastids [34]. Recently, an analysis on the Norflurazon-treated Arabidopsis seedlings using an 8200-EST microarray showed that expression of 182 genes was repressed by three-fold or more [35]. In our microarray analysis, expression of 83 genes including many photosynthetic genes was repressed by three-fold or more (Supplemental information, Table S1). Some of these photosynthetic genes were repressed in a similar pattern with those in Norflurazon-treated seedlings, indicating that the chloroplast-nucleus signaling is functional in the $p d s 3$ mutant [35]. Therefore, since carotenoid biosynthesis and initial steps of GA biosynthesis occur in plastids, it is possible that expression of the involved genes could be reduced in the $p d s 3$ mutant due to the photo-oxidative damage. Second, there might be a negative feedback mechanism in the carotenoid biosynthesis pathway. It was reported that the expression of GA5 and GA4 in leaves, stems and flower buds was noticeably inhibited by GA treatment in Arabidopsis, suggesting the existence of a negative feedback regulation in GA biosynthesis [36]. Similarly, blocking the haem branch could feedback-regulate upstream genes in the tetrapyrrole biosynthesis pathway, which in turn inhibits the chlorophyll branch, another branch in the same pathway [37]. It is interesting to note that biosyntheses of both tetrapyrrole and GAs represent branches of the carotenoid biosynthesis pathway. In this study, the expression of GGPS, PSY and $C P S$ was shown to be reduced in the $p d s 3$ mutant. GGPS and PSY are located upstream of $P D S 3$ in the carotenoid biosynthesis pathway, and CPS encodes the first enzyme in the GA biosynthesis pathway. GGPP, the common substrate for both $P S Y$ and $C P S$, is synthesized through the catalysis by GGPS. Hence, there might be a feedback mechanism in the pathway that leads to a reduced production of GAs when the function of the $P D S 3$ gene is disrupted.

\section{Acknowledgments}

This research was supported by the National Natural Science Foundation of China (Grant No. 30470172). The authors would like to thank Profs M Liu, N-S Pan, S-N Bai and S-Y Hu (College of Life Sciences, Peking University, Beijing 100871, China) for their technical assistance and valuable discussions, Mr B-L Zhang (Jiangsu Provincial 
Academy of Agricultural Sciences, Nanjing 210014, China) for help in the examination of phytohormones, and Dr K-Q Fan and Prof L-H Lai (College of Chemistry and Molecular Engineering, Peking University, Beijing 100871, China) for help in the examination of carotenoids in Arabidopsis. Special thanks go to other members of the authors' group in Peking University, Miss X-H Deng, J Ma and J Wang for growing Arabidopsis plants and Mrs P-C Liu and C Chen for helping analyze the microarray data.

\section{References}

1 Bartley GE, Scolnik PA. Plant carotenoids: pigments for photoprotection, visual attraction, and human health. Plant Cell 1995; 7:1027-1038.

2 Bendich A. Recent advances in clinical research involving carotenoids. Pure Appl Chem 1994; 66:1017-1024.

3 Koornneef M, Léon-Kloosterziel KM, Schwartz SH, Zeevaart, JAD. The genetic and molecular dissection of abscisic acid biosynthesis and signal transduction in Arabidopsis. Plant Physiol Biochem 1998; 36:83-89.

4 Andrew LP. Gibberellins in Arabidopsis. Plant Physiol Biochem 1998; 36:115-124.

5 Wettstein DV, Gough S, Kannangara CG. Chlorophyll biosynthesis. Plant Cell 1995; 7:1039-1057.

6 Chamovitz D, Sandmann G, Hirschberg J. Molecular and biochemical characterization of herbicide-resistant mutants of cyanobacteria reveals that phytoene desaturation is a rate-limiting step in carotenoid biosynthesis. J Biol Chem 1993; 268:1734817353.

7 Chamovitz D, Pecker I, Sandmann G, Boger P, Hirschberg J. Cloning a gene coding for norflurazon resistance in cyanobacteria. Z Naturforsch 1990; 45:482-486.

8 Bartley GE, Viitanen PV, Pecker I, et al. Molecular cloning and expression in photosynthetic bacteria of a soybean cDNA coding for phytoene desaturase, an enzyme of the carotenoid biosynthesis pathway. Proc Natl Acad Sci USA 1991; 88:6532-6536.

9 Hugueney P, Romer S, Kuntz M, Camara B. Characterization and molecular cloning of a flavoprotein catalyzing the synthesis of phytofluene and zeta-carotene in Capsicum chromoplasts. Eur J Biochem 1992; 209:399-407.

10 Pecker I, Chamovitz D, Linden H, Sandmann G, Hirschberg J. A single polypeptide catalyzing the conversion of phytoene to zeta-carotene is transcriptionally regulated during tomato fruit ripening. Proc Natl Acad Sci USA 1992; 89:4962-4966.

11 Mann V, Pecker I, Hirschberg J. Cloning and characterization of the gene for phytoene desaturase (PDS) from tomato (Lycopersicon esculentum). Plant Mol Biol 1994; 24:429-434.

12 Scolnik PA, Bartley GE. Phytoene desaturase from Arabidopsis. Plant Physiol 1993; 103:1475.

13 Li Z-H, Matthews PD, Burr B, Wurtzel ET. Cloning and characterization of a maize cDNA encoding phytoene desaturase, an enzyme of the carotenoid biosynthetic pathway. Plant Mol Biol 1996; 30:269-279.

14 Norris SR, Barrette TR, DellaPenna D. Genetic dissection of carotenoid synthesis in Arabidopsis defines plastoquinone as an essential component of phytoene desaturation. Plant Cell 1995; 7:2139-2149.
15 Norris SR, Shen X, DellaPenna D. Complementation of the Arabidopsis pds1 mutation with the gene encoding p-hydroxyphenylpyruvate dioxygenase. Plant Physiol 1998; 117:1317-1323.

16 Wu D-Y, Wright DA, Wetzel C, Voytas DF, Rodermel SR. The IMMUTANS variegation locus of Arabidopsis defines a mitochondrial alternative oxidase homolog that functions during early chloroplast biogenesis. Plant Cell 1999; 11:43-56.

17 Carol P, Stevenson D, Bisanz C, et al. Mutations in the Arabidopsis gene IMMUTANS cause a variegated phenotype by inactivating a chloroplast terminal oxidase associated with phytoene desaturation. Plant Cell 1999; 11:57-68.

18 Giuliano G, Bartley GE, Scolnik PA. Regulation of carotenoid biosynthesis during tomato development. Plant Cell 1993; 5:379387.

19 Corona V, Aracri B, Kosturkova G, et al. Regulation of a carotenoid biosynthesis gene promoter during plant development. Plant J 1996; 9:505-512.

20 Wetzel CM, Rodermel SR. Regulation of phytoene desaturase expression is independent of leaf pigment content in Arabidopsis thaliana. Plant Mol Biol 1998; 37:1045-1053.

21 Clough SJ, Bent AF. Floral dip: a simplified method for Agrobacterium-mediated transformation of Arabidopsis thaliana. Plant J 1998; 16:735-743.

22 Weigel D, Ahn JH, Blázquez MA, et al. Activation tagging in Arabidopsis. Plant Physiol 2000; 122:1003-1014.

23 Wagner DB, Furnier GR, Saghai-Maroof MA, et al. Chloroplast DNA polymorphisms in lodgepole and jack pines and their hybrids. Proc Natl Acad Sci USA 1987; 84:2097-2100.

24 Liu YG, Huang N. Efficient amplification of insert end sequences from bacterial artificial chromosome clones by thermal asymmetric interlaced PCR. Plant Mol Biol Reporter 1998; 16:175181.

25 Qin G, Kang D, Dong Y, et al. Obtaining and analysis of flanking sequences from T-DNA transformants of Arabidopsis. Plant Sci 2003; 165:941-949.

26 Sandmann G, Albrecht M. Accumulation of colorless carotenes and derivatives during interaction of bleaching herbicides with phytoene desaturation. Z Naturforsch 1990; 45:487-491.

27 Ma L, Li J, Qu L, et al. Light control of Arabidopsis development entails coordinated regulation of genome expression and cellular pathways. Plant Cell 2001; 13:2589-2607.

28 Nepi M, Ciampolini F, Pacini E. Development and ultrastructure of Cucurbita pepo nectaries of male flowers. Ann Bot 1996; 78:95-104.

29 Alonso JM, Stepanova AN, Leisse TJ, et al. Genome-wide insertional mutagenesis of Arabidopsis thaliana. Science 2003; 301:653-657.

30 Estevez JM, Cantero A, Romero C, et al. Analysis of the expression of CLA1, a gene that encodes the 1 - deoxyxylulose 5 - phosphate synthase of the $2-\mathrm{C}$ - methyl - D - erythritol - 4 - phosphate pathway in Arabidopsis. Plant Physiol 2000; 124:95104.

31 Schoof H, Zaccaria P, Gundlach H, et al. MIPS Arabidopsis thaliana Database (MAtDB): an integrated biological knowledge resource based on the first complete plant genome. Nucleic Acids Res 2002 30:91-93.

32 Motohashi R, Nagata N, Ito T, et al. An essential role of a TatC homologue of a $\mathrm{pH}$-dependent protein transporter in thylakoid membrane formation during chloroplast development in $\mathrm{Ara}$ - 
bidopsis thaliana. Proc Natl Acad Sci USA 2001; 98:1049910504.

33 Mayfield SP, Taylor WC. Carotenoid-deficient maize seedlings fail to accumulate light-harvesting chlorophyll $\mathrm{a} / \mathrm{b}$ binding protein (LHCP) mRNA. Eur J Biochem 1984; 144:79-84.

34 Surpin, M., Larkin, R.M. and Chory, J. Signal transduction between the chloroplast and the nucleus. Plant Cell 2002; 14: S327-S338.

35 Strand A, Asami T, Alonso J, Ecker JR, Chory J. Chloroplast to nucleus communication triggered by accumulation of Mg-protoporphyrinIX. Nature 2003; 421:79-83.

$36 \mathrm{Xu}$ Y-L, Li L, Gage DA, Zeevaart JAD. Feedback regulation of GA5 expression and metabolic engineering of gibberellin levels in Arabidopsis. Plant Cell 1999; 11:927-935.

37 Terry MJ, Kendrick RE. Feedback inhibition of chlorophyll synthesis in the phytochrome chromophore-deficient aurea and yellow-green-2 mutants of tomato. Plant Physiol 1999; 119:143152.

(Supplementary information is linked to the online version of the paper on the Cell Research website.) 